\title{
Influence of Base Pressure on FeMn Exchange Biased Spin-valve Films
}

\author{
M. Mao, C. Cerjan, B. Law, F. Grabner, S. Vaidya
}

This article was submitted to the $44^{\text {th }}$ Annual Conference on Magnetism and Magnetic Materials, San Jose, CA, November 15-18, 1999

\section{August 13, 1999}

U.S. Department of Energy






\section{DISCLAIMER}

This document was prepared as an account of work sponsored by an agency of the United States Government. Neither the United States Government nor the University of California nor any of their employees, makes any warranty, express or implied, or assumes any legal liability or responsibility for the accuracy, completeness, or usefulness of any information, apparatus, product, or process disclosed, or represents that its use would not infringe privately owned rights. Reference herein to any specific commercial product, process, or service by trade name, trademark, manufacturer, or otherwise, does not necessarily constitute or imply its endorsement, recommendation, or favoring by the United States Government or the University of California. The views and opinions of authors expressed herein do not necessarily state or reflect those of the United States Government or the University of California, and shall not be used for advertising or product endorsement purposes.

This is a preprint of a paper intended for publication in a journal or proceedings. Since changes may be made before publication, this preprint is made available with the understanding that it will not be cited or reproduced without the permission of the author.

This report has been reproduced directly from the best available copy.

Available to DOE and DOE contractors from the Office of Scientific and Technical Information

P.O. Box 62, Oak Ridge, TN 37831

Prices available from (423) 576-8401 http:/ / apollo.osti.gov/bridge/

Available to the public from the National Technical Information Service U.S. Department of Commerce 5285 Port Royal Rd., Springfield, VA 22161 http://www.ntis.gov/

\section{OR}

Lawrence Livermore National Laboratory Technical Information Department's Digital Library http://www.llnl.gov/tid/Library.html 


\title{
Influence of Base Pressure on FeMn Exchange Biased Spin-valve Films
}

M. Mao, C. Cerjan, B. Law, F. Grabner and S. Vaidya

Lawrence Livermore National Laboratory, Livermore, CA 94550

\begin{abstract}
Spin-valve films of structure $\mathrm{NiFeCo} / \mathrm{Co} / \mathrm{Cu} / \mathrm{NiFeCo}(\mathrm{Co}) / \mathrm{FeMn} / \mathrm{Cu}$ were deposited on Si substrates by DC planetary magnetron sputtering techniques. The influence of base pressure, $P_{b}$, on spin-valve properties was studied by varying $P_{b}$ over two decades from $3 \times 10^{-8}$ to $7 \times 10^{-6}$ Torr. The GMR ratio show a slight increase with increasing $P_{b}$ until a large decrease occurs at $P_{b}>3.3 \times 10^{-6}$ Torr. Exchange bias field and blocking temperature remain constant in the base pressure range between $3 \times 10^{-8}$ and $5 \times 10^{-7}$ Torr before a large reduction begins. An upper bound base pressure, ${ }^{4} \mathrm{P}_{\mathrm{b}} \approx 5 \times 10^{-7}$ Torr, is noted from the data, above which significant performance modification begins. The degradation in exchange bias field and blocking temperature, in particular, in spin-valve films using a NiFeCo pinned layer, is the result of deterioration in the crystallographic texture and can be understood due to the contamination both at the ferromagnetic/antiferromagnetic interface and in the bulk of FeMn layer.
\end{abstract}




\section{AS-08}

\section{Introduction}

The control of thin layer thickness and physical properties becomes a major concern for magnetoresistive read sensor manufacturing as areal recording density increases. Since the specific surface area increases with decreasing layer thickness, thin layer formation becomes more vulnerable to contamination. Previous studies on ferromagnetic/antiferromagnetic (F/AF) bilayers $[1,2]$ have suggested that Ultra-High Vacuum (UHV) processes should be used to improve exchange biasing. UHV processes are, however, not very practical in a highly productive manufacturing environment in terms of consistent process control and throughput. This study attempts to determine an upper bound for system base pressure when impurity induced degradation occurs and to understand the performance modification of spin-valve films. FeMn exchange biased spin-valve films were chosen to study because FeMn is a well-known antiferromagnetic material that is very vulnerable to corrosion and impurity contamination.

\section{Experimental methods}

Two sets of spin-valve films, $\mathrm{NiFeCo} 40 \AA / \mathrm{Co} 10 \AA / \mathrm{Cu} 23 \AA / \mathrm{NiFeCo}(\mathrm{Co}) 20 \AA / \mathrm{FeMn} 100 \AA / \mathrm{Cu} 20 \AA$, were deposited on 4" Si(001) substrates by DC planetary magnetron sputtering techniques. A 40 Oc aligning magnetic field was applied during the film depositions. Spin-valve films with graded Cu layer thickness, $\mathrm{NiFeCo} 50 \AA / \mathrm{Cu}\left(\mathrm{t}_{\mathrm{Cu}}\right) \AA / \mathrm{NiFeCo} 20 \AA / \mathrm{FeMn} 100 \AA / \mathrm{Cu} 20 \AA$, were also prepared. The Cu layer thickness, $\mathbf{t}_{\mathrm{Cu}}$, was varied almost linearly from 18 to $38 \AA$ across the radius of a single 4 " Si substrate. The influence of base pressure, $P_{b}$, on spin-valve physical properties was studied by varying $P_{b}$ over two decades from $3 \times 10^{-8}$ to $7 \times 10^{-6}$ Torr. A SHB-109 loop tracer and a quasistatic tester were used to determine GMR value, $\Delta R / R$, exchange bias field, $H_{e x}$, and interlayer exchange field, $H_{i n}$, of the spin-valve films. The temperature dependence of $\mathrm{H}_{\mathrm{ex}}$ for selected films was measured using a DMS vibrating sample magnetometer. The blocking temperature, $T_{B}$, was then determined by fitting $H_{e x}$ vs. T curves to a power law, $H_{e x} \propto\left(T_{B}-T\right)^{\varepsilon}$. Xray diffraction and rfflectivity measurements using $\mathrm{Cu} \mathrm{K} \alpha$ radiation were also performed for film structural characterizations. 


\section{Results and Discussion}

Values of $\Delta \mathrm{R} / \mathrm{R}-7.5 \%$ and $6.5 \%$ were measured from spin-valve films using Co and NiFeCo as the pinned layers, respectively. Films using a NiFeCo pinned layer exhibit a relatively large $H_{\text {ex }}$ value $\sim 260$ Oe, as compared to a $\mathrm{H}_{\mathrm{ex}}$ value $\sim 200$ Oe for those films using a Co pinned layer. Both $\Delta \mathrm{R} / \mathrm{R}$ and $\mathrm{H}_{\mathrm{ex}}$ change as a function of $P_{b}$, as shown in Fig. 1a. A slight increase in $\Delta R / R$, to $7.8 \%$ and $7 \%$ for the films using a Co and a NiFeCo pinned layer, respectively, is seen with increasing $\mathrm{P}_{\mathrm{b}}$ from $3 \times 10^{-8}$ Torr until a significant decrease appears at $P_{b}>3 \times 10^{-6}$ Torr. The decrease in $\Delta R / R$ is accompanied by an increase in sheet resistance, $R_{s}$ indicative of impurity contamination. On the other hand, $\mathrm{H}_{\mathrm{ex}}$ remains almost constant as $P_{b}$ is increased, as shown in Fig. 1b, before a significant reduction in $H_{e x}$ is observed at $P_{b}>5 \times 10^{-7}$ Torr. Films using a NiFeCo pinned layer show almost a factor of 2.5 greater reduction in $\mathrm{H}_{\mathrm{ex}}$ than those using a Co pinned layer over a rangc of $P_{b}$ from $3 \times 10^{-7}$ to $1 \times 10^{-6}$ Torr. The interlayer exchange field, $H_{\text {in }}$, retains its value of about 10 Oe with increasing $P_{b}$ up to $3.3 \times 10^{-6}$ Torr before it starts to decrease.

The influence of base pressure on the physical properties of spin-valve films is more definitively illustrated by the films with graded $\mathrm{Cu}$ layer thickness. Three films of structure $\mathrm{NiFeCo} 50 \AA / \mathrm{Cu}\left(\mathrm{t}_{\mathrm{Cu}}\right) \AA$ / $\mathrm{NiFeCo} 20 \AA / \mathrm{FeMn} 100 \AA / \mathrm{Cu} 20 \AA$ were deposited at three different base pressures, $7.8 \times 10^{-8}, 3.3 \times 10^{-7}$ and $1.6 \times 10^{-6}$ Torr, respectively. In Fig. 2 , the dependence of physical properties on $t_{\mathrm{Cu}}$ is displayed as a function of base pressure. While there is little difference in the sheet resistance between these three films, the film deposited at a higher base pressure does show relatively large values of $\Delta R / R$, as shown in Fig. 2a, which corresponds to slightly higher values of interlayer exchange field (Fig. 2b). The degradation of exchange bias field with inceasing base pressure is also clearly demonstrated in Fig. $2 \mathrm{~b}$.

The temperature dependence of $H_{c x}$ for the FeMn exchange biased spin-valve films can be well described by a power law, $H_{c x} \propto\left(T_{B}-T\right)^{\varepsilon}$, as shown in Fig. 3a, where the exponent, $\varepsilon$, varies with increasing $P_{b}$ from 1.41 to 1.75 . The blocking temperature, $T_{B}$, is determined to be $140 \mathrm{C}$ for the films deposited at $P_{b} \leq 4 \times 10^{-7}$ Torr. The relatively low $T_{B}$ value, as compared to the $150 \mathrm{C}$ value commonly observed in FeMn cxchange biased spin-valve films, is probably due to the non-stoichiometric composition of the FeMn films, which was determined to be $\mathrm{Fe}_{55} \mathrm{Mn}_{45}$ by energy dispersive $\mathrm{X}$-ray analysis techniques. Fig. $3 \mathrm{~b}$ shows blocking 


\section{AS-08}

temperature as a function of base pressure. $T_{B}$ remains almost constant with increasing $P_{b}$. A large reduction in $T_{B}$ occurs at $P_{b}>5 \times 10^{-7}$ Torr.

It follows from the results described above that there exists an upper bound for the system base pressure, ${ }^{u} \mathrm{P}_{\mathrm{b}} \approx 5 \times 10^{-7}$ Torr. A further increase in $\mathrm{P}_{\mathrm{b}}$ above ${ }^{\mathrm{u}} \mathrm{P}_{\mathrm{b}}$ results in a significant performance modification in FeMn exchange biased spin-valve films..

X-ray diffraction and reflectivity measurements were preformed to understand the modification in film magnetic properties. Fig. 4 shows $\mathrm{x}$-ray diffraction patterns for the films using a Co pinned layer deposited at different base pressures. All films exhibit exclusively (111) texture. Because of the closeness of the lattice constants between $f c c$ FeMn $(\sim 3.67 \AA)$ and $\mathrm{NiFeCo} / \mathrm{Co} / \mathrm{Cu} / \mathrm{Co}(\sim 3.60 \AA)$ [3], and limited instrumental resolution, only one diffraction peak can be resolved, which is the superposition of $f c c$ FeMn (111) and $\mathrm{NiFeCo} / \mathrm{Co} / \mathrm{Cu} / \mathrm{Co}$ (111) peaks. The (111) diffraction peak was analyzed by fitting the data to a Lorentzian line shape. The peak intensity thus derived decreases with increasing $P_{b}$, as clearly indicated in Fig. 5a. Noticeable deterioration in (111) texture begins at $P_{b}>5 \times 10^{-7}$ Torr. The diffraction peak broadens and shifts to large angles with increasing $P_{b}$ to above $5 \times 10^{-7}$ Torr, as shown in Fig. $5 \mathrm{~b}$. The reduction in (111) diffraction peak intensity can bc mainly attributed to the deterioration of the FeMn layer so that the relative contribution from FeMn layer to the integrated intensity of (111) diffraction peak decreases and the diffraction peak shifts to a high angular position as $\mathbf{P}_{\mathbf{b}}$ increases. The broadening in diffraction peak is the result of increased symmetry in the contributions to the diffraction peak intensity from $\mathrm{FeMn}$ and $\mathrm{NiFeCo} /$ $\mathrm{Co} / \mathrm{Cu} / \mathrm{Co}$ layers and reduced grain sizes at higher base pressures. This degradation is responsible for the significant reduction in exchange bias field and blocking temperature, as shown in Fig. $1 \mathrm{~b}$ and Fig. $3 \mathrm{~b}$.

Fig. 6 shows x-ray reflectivity of the films using a NiFeCo pinned layer. There exists virtually no difference between reflectivity curves of the films deposited at various base pressures over two decades. The reflectivity is reasonably well described by a simple model, as illustrated by the solid lines in Fig. 6 for selected films. The results from this analysis indicate a standard deviation $\leq 1 \AA$ of component layer thicknesses and interfacial roughnesses within $2 \AA$ deposited for the films deposited over a base pressure range from $3 \times 10^{-8}$ to $3 \times 10^{-6}$ Torr. The integrity of the layered structure remains with increasing base pressure up to $3 \times 10^{-6}$ Torr, which is the main reason for the stability of GMR ratio of the films deposited at $\mathrm{P}_{\mathrm{b}} \leq 3 \times 10_{-6}$ Torr. 


\section{AS-08}

A reduction in grain size by about $50 \AA$ along the growth direction and $30 \AA$ by increasing $\mathrm{P}_{\mathrm{b}}$ from $1 \times 10^{-7}$ to $8 \times 10^{-7}$ Torr was previously observed in $\mathrm{Co} / \mathrm{Cu}$ multilayers [4]. In the present case, there appears no obvious improvement in interfacial smoothness with increasing base pressure up to $\sim 3 \times 10^{-6}$ Torr, as expected from the grain size reduction $[5,6]$. To the contrary, the results from films with graded $\mathrm{Cu}$ layer thickness suggest a slight increase in interfacial roughness as implicated by the slight increase in the interlayer exchange field with increasing base pressure (Fig. 2b). To account for the increase in $\Delta R / R$ at $\mathrm{Pb} \leq 3 \times 10^{-6}$ Torr, a possible mechanism would be electron specular scattering at the interface between the $\mathrm{NiFeCo}$ free layer and the Si substrate since a nano oxide layer may have formed during the first few second deposition at a high system base pressure. Further investigation will be necessary to clarify this effect.

\section{Conclusions}

The influence of base pressure on the physical properties of FeMn-exchange biased spin-valve films has been systematically studied. The spin-valve performance remains stable with increasing base pressure until an upper bound, " ${ }^{\mathrm{b}} \approx 5 \times 10^{-7}$ Torr, is reached. Above ${ }^{\mathrm{u}} \mathrm{P}_{\mathrm{b}}$, significant performance modification begins. The degradation in exchange bias field and blocking temperature, in particular, in spin-valve films using a $\mathrm{NiFeCo}$ pinned layer, is the result of deterioration in crystallographic quality and is caused by the impurity contamination both at the ferromagnetic/antiferromagnetic interface and in the bulk of the FeMn layer.

\section{Acknowledgment}

We thank C.-Y. Hung, L. Miloslavsky and S. Bargotrafrom from Read-Rite Corporation for their valuable assistance in the VSM, high angle x-ray diffraction and EDX measurements.

The work was performed under the auspices of the U.S. Department of Energy by Lawrence Livermore National Laboratory under contract No. W-7405-Eng-48.

\section{References}

[1] A. Tanaka, Y. Shimizu, H. Kishi, K. Nagasaka, H. Kanai and M. Oshiki, IEEE Trans. Mag. 35, 700

[2] M. Tsunoda, K. Uneyama, T. Suzuki, K. Yagami and M. Takahashi, J. Appl. Phys. 85, 4919 (1999). 
[3] L. Tang, D.E. Lauphlin and S. Ganggopadhyay, J. Appl. Phys. 81, 4906 (1997).

[4] M. Mao, unpublished.

[5] M. Mao, C. Cerjan, M. Gibbons, B. Law, F. Grabner, S. Vernon and M. Walls, IEEE Trans. Mag. 34, 915 (1989).

[6] S. Miura, D. Takahashi, M. Tsunoda and M. Takahashi, J. Appl. Phys. 34, 934 (1998).

\section{Figure Captions}

FIG. 1 (a) Sheet resistance, $R_{s}$, GMR ratio, $\Delta R / R$, (b) exchange bias field, $H_{c x}$ and interlayer exchange field, $H_{\text {in }}$, as a function of hase pressure for spin-valve films of structure NiFeCo40 $\AA / C o 10 \AA / C u 23 \AA$ / $\mathrm{P} 20 \AA / \mathrm{FeMn} 100 \AA / \mathrm{Cu} 20 \AA$, where $\mathrm{P}$ is either a NiFeCo (triangle) or a Co (diamond) pinned layer. The lines are guides for the eye.

FIG. 2 Dependence of physical properties on $\mathrm{Cu}$ layer thickness for three spin-valve films $\mathrm{NiFeC0} 50 \AA / \mathrm{Cu}\left(\mathrm{t}_{\mathrm{Cu}}\right) \AA / \mathrm{NiFeCo} 20 \AA / \mathrm{FeMn} 100 \AA / \mathrm{Cu} 20 \AA$ with graded $\mathrm{Cu}$ layer thickness deposited at different base pressures: 0.78 (diamond), 3.3 (square) and 16 (triangle) $\times 10^{-7}$ Torr. The lines are guides for the eye.

FIG. 3 (a) Temperature dependence of exchange bias field for spin-valve films using a NiFeCo pinned layer deposited at different base pressures, where the lines are the fits to the power law; (b) base pressure dependence of biocking temperature.

FIG. 4 X-ray diffraction patterns for spin-valve films of structure NiFeCo $40 \AA / \mathrm{Co} 10 \AA / \mathrm{Cu} 23 \AA / \mathrm{Co} 20 \AA$

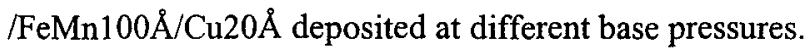

FIG. 5 (a) (111) x-ray diffraction peak intensity, (b) peak position, $2 \theta^{(111)}$, and peak full width at half maximum, $\Delta 2 \theta^{(11)}$, as a function of base pressure for spin-valve films of structure NiFeCo $40 \AA /$



FIG.6 X-ray reflectivity as a function of base pressure for spin-valve films of $\mathrm{NiFeC0} 40 \AA / \mathrm{Co} 10 \AA / \mathrm{Cu} 23 \AA / \mathrm{NiFeCo} 20 \AA / \mathrm{FeMn} 100 \AA / \mathrm{Cu} 20 \AA$. The solid lines are the fits to the model for data from selected films. 

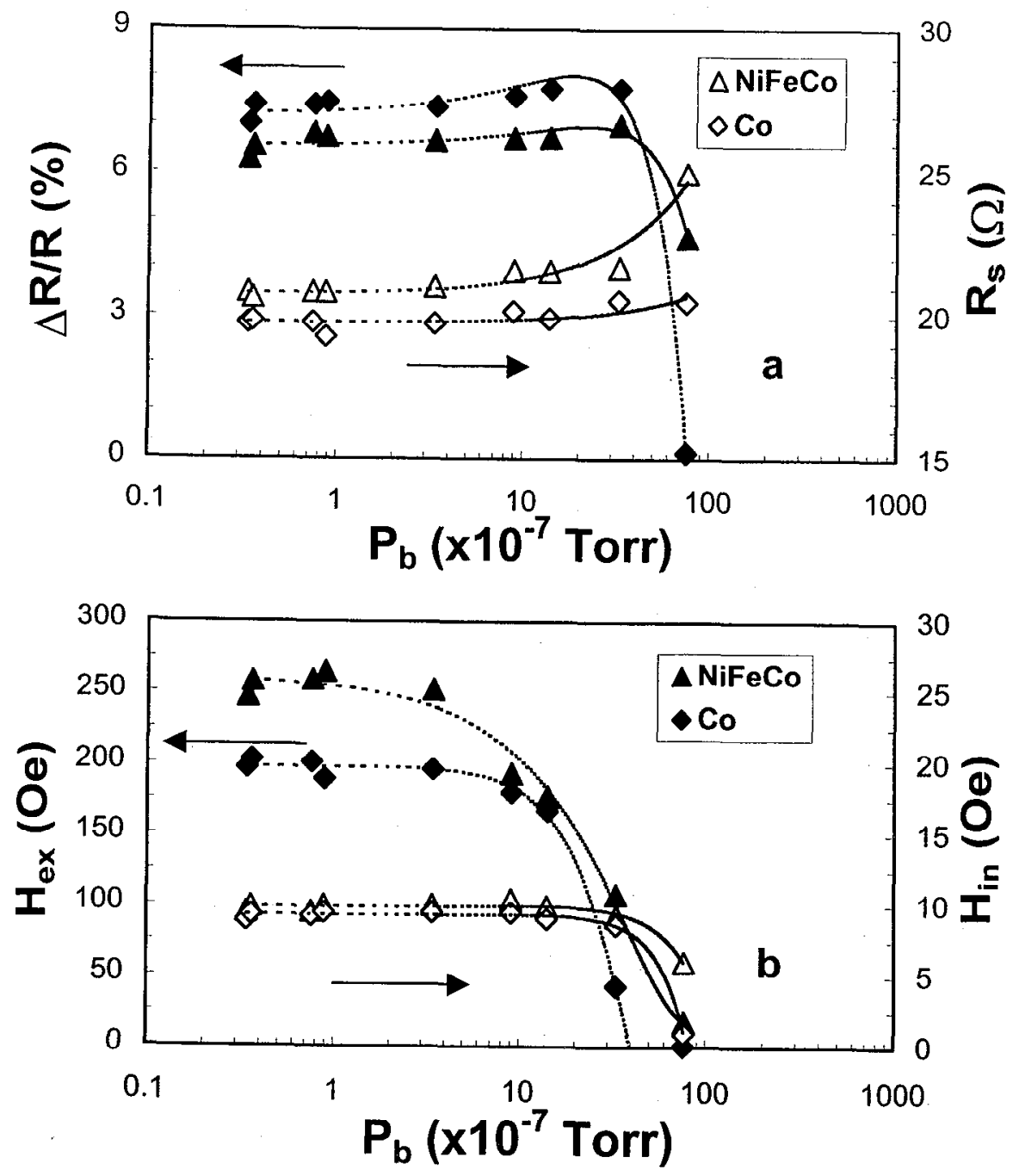

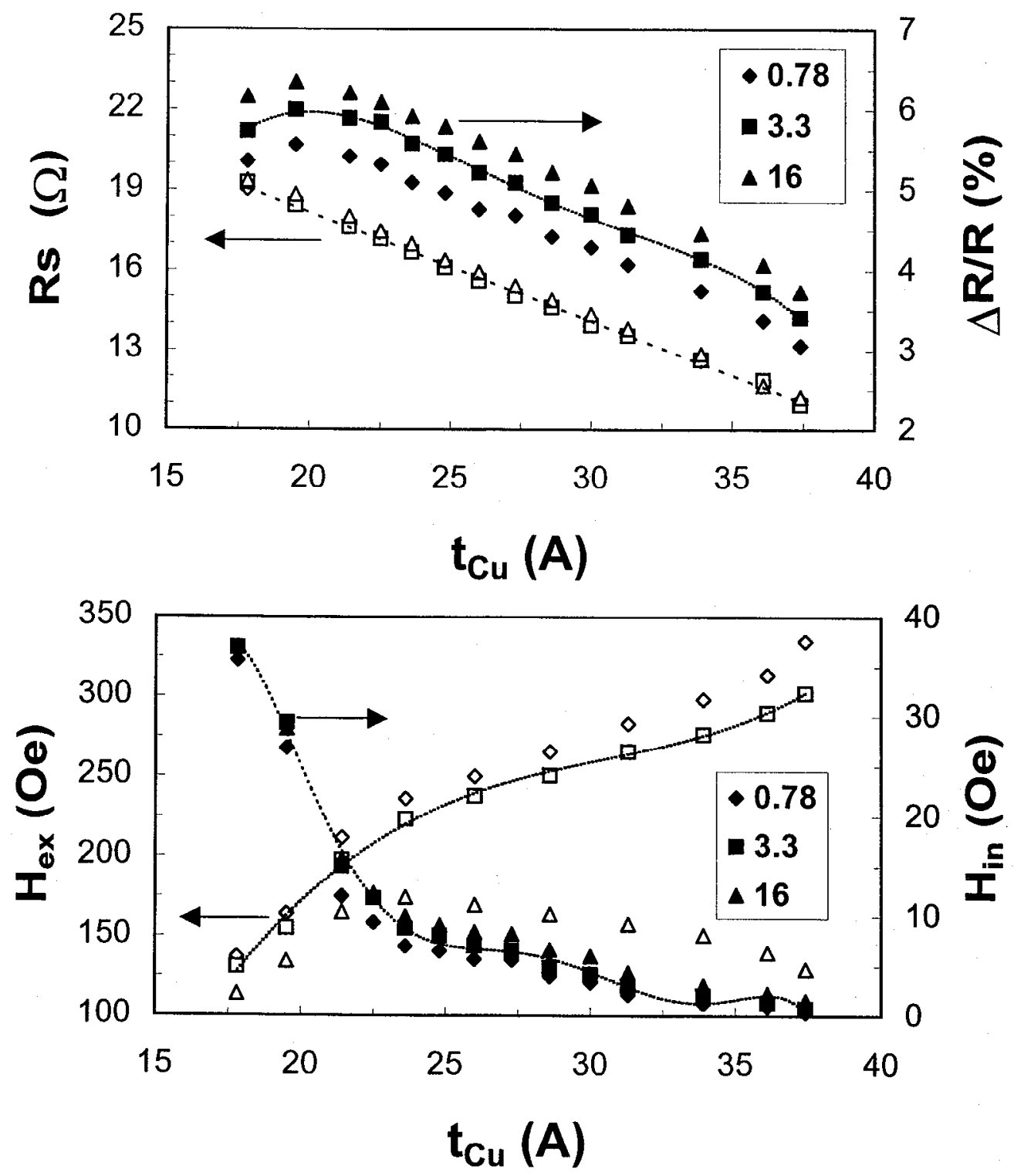

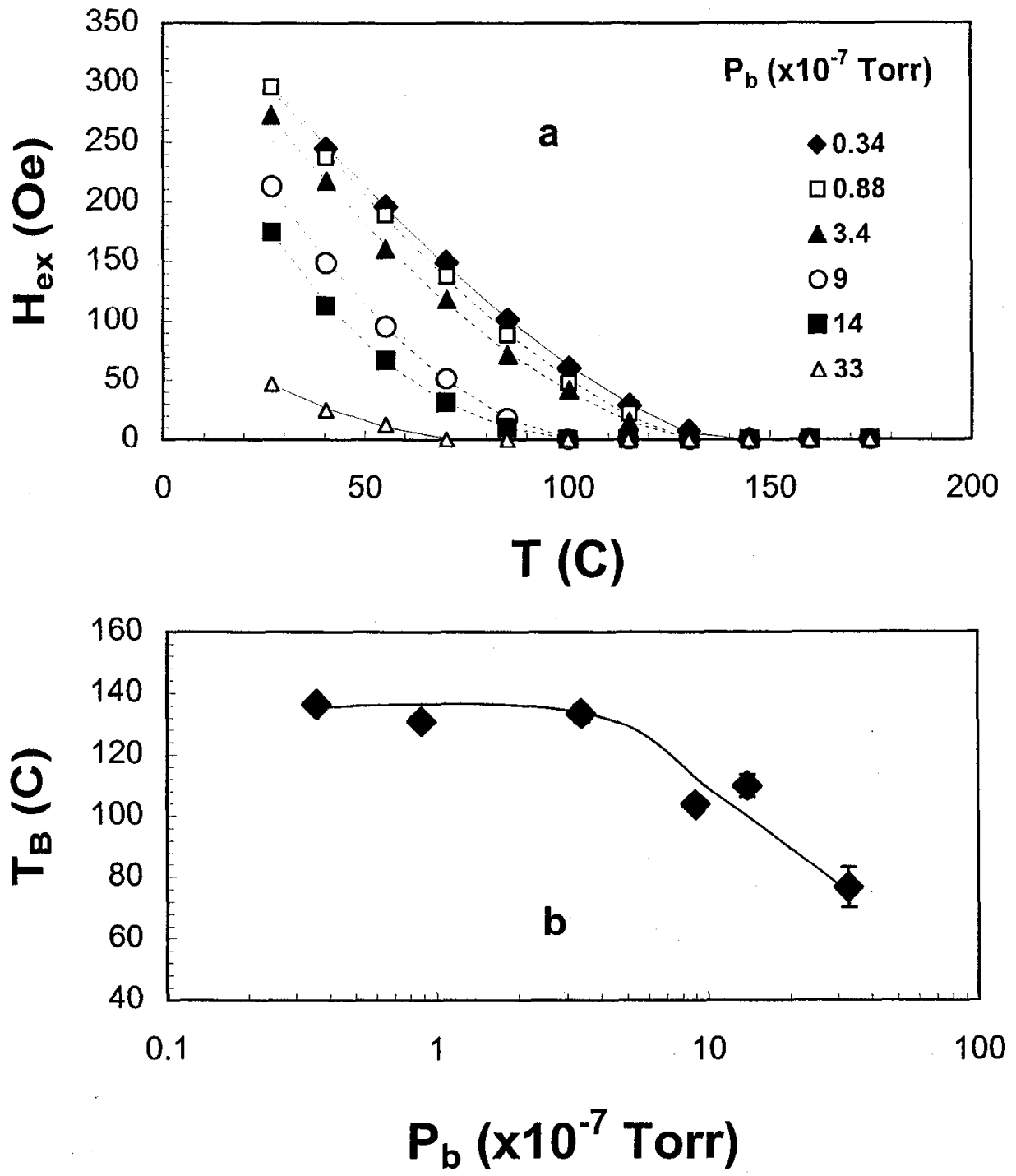






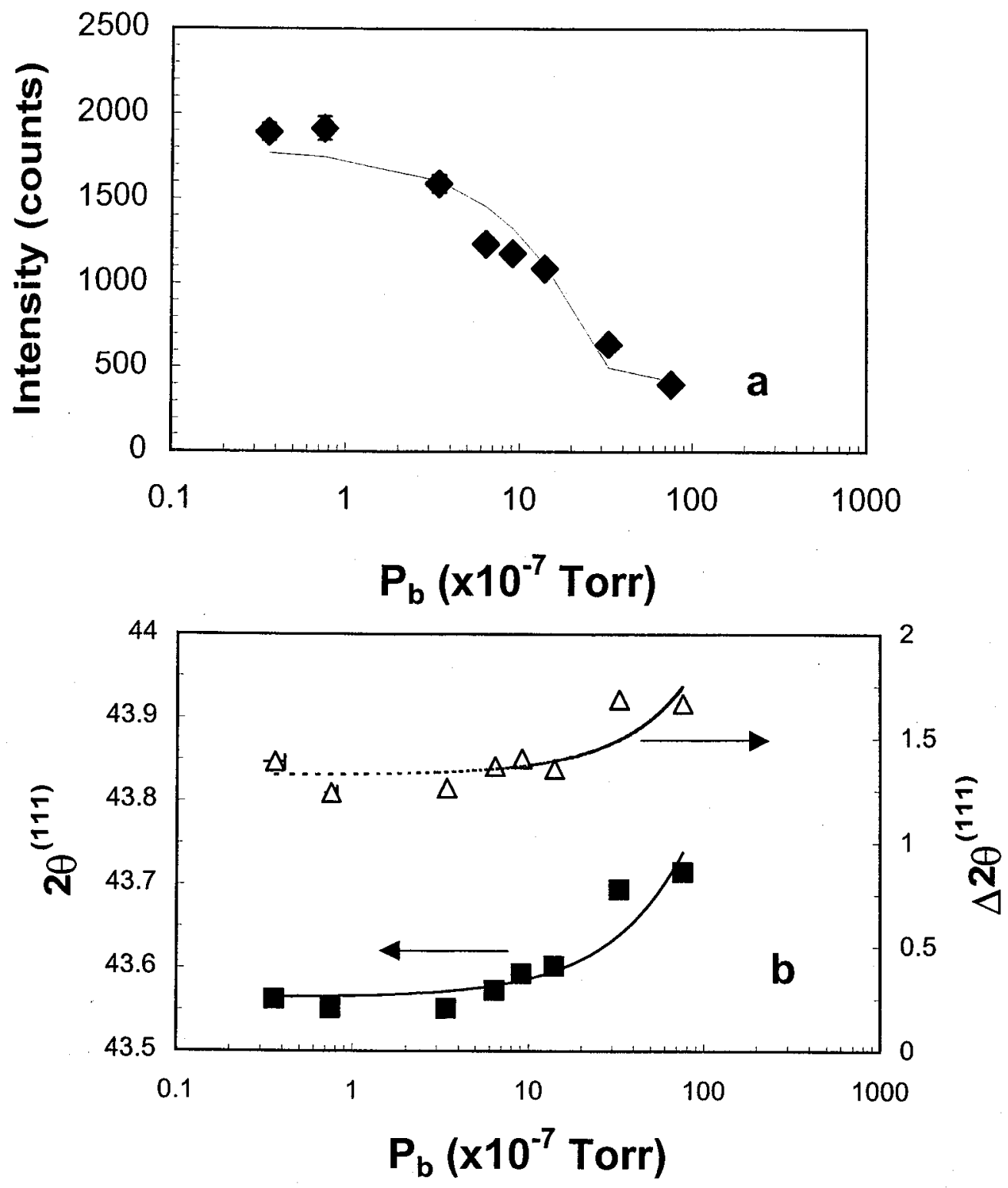

Fig. 5

M. Mao et al. 




